\title{
Louis de Broglie's wave-particle duality: from textbooks' blackboxes to a chain of reference presentation
}

\author{
Nathan Willig Lima*1@, Matheus Monteiro Nascimento@ Cláudio Cavalcanti@ ${ }^{1 @}$, Fernanda \\ Ostermann 10 \\ ${ }^{1}$ Universidade Federal do Rio Grande do Sul, Instituto de Física, Porto Alegre, RS, Brasil.
}

Received on June 07, 2019. Revised on October 31, 2019. Accepted on October 31, 2019

\begin{abstract}
We discuss the proposition of wave-particle duality made by Louis de Broglie in the period of 1922-1924 as well as the narratives of undergraduate Physics textbooks that introduce this topic. After a brief discussion on the nature of scientific reality, we point out the main theories, assumptions and techniques adopted by de Broglie to achieve the relation between momentum and wavelength. We use these elements as analytical categories, observing which of them are still present in four popular undergraduate introductory textbooks. Our results show that none of the textbooks used de Broglie's original strategy (mathematical derivation), treating his ideas as postulates. Moreover, we show that only few elements of de Broglie's original presentation are still commented by textbooks, which treat de Broglie's ideas as purely speculative, reinforcing the "genius myth". In the sequence, we propose a didactic alternative, which we call a "chain of reference presentation". In this strategy, we highlight that all scientific knowledge is supported by previous theories and it works as the ground of future scientific improvements. With this perspective, we perform a didactic derivation of de Broglie's momentum-wavelength relation (based on the original papers and on the specialized literature) and we introduce empirical and theoretical works that may be studied from it.
\end{abstract}

Keyword: Quantum Mechanics, Textbooks, Epistemology.

\section{Introduction}

Physicists consider Quantum Physics (QP) the most complete theory to explain the structure of matter, delivering a high degree of precision in its experimental predictions. During the 20th century, its development led to the formation of new areas of Physics such as Quantum Field Theory [1], [2], Quantum Optics [3], [4] Quantum Information [5], Quantum Thermodynamics [6], Quantum Gravitation [7], among others, as well as to the development of different technological applications in the aforementioned areas, such as Nuclear Engineering and Semiconductor Physics, but also in Medicine [8] and much of nanosciences [9]. In addition, the quantum mysticism movement has broadly spread QP terminology through idealistic interpretations of reality [10] - making its elements part of non-scientist vocabulary.

In general, one can think that QP refers to two major movements in Physics: the first involves studies on the nature of radiation and the structure of matter in the first half of the 20th century, culminating in the proposition of Schrödinger's Equation and Heisenberg's matrix physics; and the second involves the isolation of individual quantum objects, quantum entanglement, and Quantum Information Theory, which were developed in the second half of the twentieth century and in the twenty-first century [11].

*Correspondence email address: nathan.lima@ufrgs.br
In the context of the first movement, the proposition of wave-particle duality can be thought of as a central theme, or even as the only mystery of QP [12]. A first suggestion about it appears in an article by Einstein in 1909 about the quantum [13] with a different conception of his seminal paper on the theme [14], but it was just formalized and generalized by de Broglie in the beginning of the twenties ${ }^{1}$ [15]. Along the development of Quantum Physics, however the meaning of wave-particle duality has substantially varied - attributing different ontological natures to the particle and to the wave in the dual phenomena [16]-[19]. Schrödinger [20], for instance, proposed that radiation should be considered as a real material wave propagating in the phase space, while its particle-like behavior should be attributed as a consequence of the quantized atom (a vision shared by Planck),

\footnotetext{
In his first papers about the nature of light, Einstein defended that radiation should be constituted by localized quanta in space in opposition to its description as continuous wave [14]. In 1909, however, he computed the statistical fluctuations of energy in the black-body radiation, achieving a term corresponding to a continuous radiation and a term corresponding to a quantized radiation. Einstein concluded that "I more or less imagine each such singular point as being surrounded by a field of force which has essentially the character of a plane wave and whose amplitude decreases with the distance from the singular point." [13]. Despite of this suggestion, Einstein has not achieved any formalized theory for dual radiation. That is why there is a historiographic controversy about considering de Broglie or Einstein as the "founder" of wave particle duality [15] .
} 
even contesting Compton's explanation on the collision between photons and electrons [21]. Born [22], in the other hand, considered that the "wave" is not something material, in nature, but merely an epistemic tool, an instrument that informs us about our own knowledge, while the particle is what is real. Almost at the same time, Bohr [23] proposed the Complementarity Principle, stating that wave-like and particle-like phenomena are complementary aspects of the same entity, not being able to be observed mutually. In this proposition, the experimental set up is what determines the appearance of one or another aspect.

A possible way to escape this ontological controversy in QP Education would be to follow the suggestion made by some researchers of relativistic quantum mechanics: there are no particles, only fields [24], [25]. However, the wave-particle duality is still a major theme in contemporary Quantum Physics historiography [15], [26] and its historical and conceptual relevance also motivates contemporary research in Quantum Physics Teaching [27]-[29], besides of being a common subject in Quantum Physics courses in undergraduate and high-school levels [18], [30]. We highlight that this topic may be approached in different trends of Quantum Physics Teaching such as the development of didactic experiments [31]-[36], the use of metaphors [37], [38], production of tutorials [29], [39], [40], the use of computational simulations [41]-[46], the analysis of didactic textbooks [47], [48], and so on .

In the Brazilian context, as in many other countries, the research on Quantum Physics textbooks and on the production of didactic material is especially important since future physicists and teachers of Physics are usually introduced to this concept in basic Physics courses (commonly in the fourth semester of the undergraduate course) or in disciplines like Introduction to Quantum Physics (or Modern Physics), in which the studies on radiation of the first two decades of the twentieth century are presented through textbooks and not the original papers.

Textbooks, in this sense, are understood as a material element of the so called Science Education Culture . [49], [50], being a mediator (or a translator) between the scientific gender (original papers) and scientists and science teachers in training. This is why textbooks are not only important to the pedagogic context but to Science itself, as it was recognized by Thomas Kuhn in The Structure of Scientific Revolution [51]. According to Kuhn, textbooks are responsible for structuring scientific disciplines and determining the methods and problems that future scientists should be circumscribed to. This implies, in the kuhnian description, that textbooks perpetuate scientific knowledge by representing it as a collection of evident truths [52]. In the other hand, Kuhn also pointed out that textbooks authors tend to erase controversy, crisis, historical contingency and so on, which demotivated the research on textbooks under a historiographical approach [53].

According to Badino and Navarro [50], however, the interest about the role of textbooks in science history have increased since the late nineties. The authors mention, for instance the Science Education special topic on textbook in the scientific periphery [54] and David Kaiser's work on scientific pedagogy [55]. Moreover, the authors argue that the analysis of textbooks written in the period of scientific crisis, such as the quantum revolution, has shown that they cannot be seen as a "mausoleum of scientific truths" (as Kuhn would describe it), but that they contributed actively to the scientific development. Furthermore, the narrative of undergraduate textbooks sometimes is so relevant that is more diffused than the original paper narrative [18].

In this scenario, we have two goals. The first one is to contribute to the analysis of contemporary undergraduate textbooks that introduce de Broglie's particle-wave duality by evaluating how they dialogue with de Broglie's original papers. The second one is to propose a different didactic strategy, which stresses the original techniques, theories and assumptions that de Broglie used to articulate his proposition as well as the empirical and theoretical improvements that were allowed from it, based on de Broglie original papers and the specialized literature. More specifically, in the first part of the paper we propose to answer three questions a) What is the meaning attributed to wave-particle duality by Louis de Broglie in the period of 1922-1924? b) Which tools, techniques and theories did he use to support his proposition? c) How do introductory undergraduate textbooks describe Louis de Broglie's proposition and which elements (tools, techniques and theories) they mention to support it?

To answer questions "a" and "b", we analyzed de Broglie's three original papers written from 1922 to 1924 [56]-[58] and interpretations offered by secondary sources [15], [59], [60]. To answer question "c", we analyzed four contemporary higher education textbooks used in the Brazilian Physics introductory disciplines [61]-[64].

In order to present the results of our analysis we structured the paper in the following sequence. In section 2 , we present the theoretical background that supported our analysis of the textbooks and we introduce the adopted methodological trajectory. In section 3, we introduce the mains aspects of de Broglie's original articulation and use them as analytical categories to perform textbook analysis. In section 4 , we propose a didactic narrative that highlights de Broglie's theoretical backgrounds, assumptions and techniques. By doing so, we show a possible way to derive his famous relation between momentum and wavelength, in dialogue with de Broglie original conceptions and with secondary literature, and we discuss how de Broglie's proposition allows the formation of new empirical and theoretical works.

\section{Theoretical Framework: Science Dialectic Movements and Scientific Reality}

The conception about what science is and how it works is source of a profound and complex debate in the area 
of Philosophy and Sociology of Science [65] as well as the discussion about what reality is and how science relates to this reality [66]. In the present work, we mainly acknowledge some of Gaston Bachelard's considerations about epistemology and ontology [67], [68] in dialogue with other contemporary trends.

The scientific thinking, to Bachelard, is strongly attached to empiricism and rationalism in a rather complementary conception instead of a dualist perspective:

One would misunderstand us if he sees in it a simple recognition of dualism. Just the opposite, the epistemological polarity is to us the proof that each of the philosophical doctrines that we call empiricism and rationalism is the effective complement of the other. One finishes the other. To think scientifically is to be in the intermediary epistemological field between theory and practice, between mathematics and experience. To know scientifically a natural law is to know it simultaneously as phenomenon and number $[67, \mathrm{p} .5]$.

These two epistemological poles interact in a dialectic movement: "empiricism must be understood and rationalism must be applied" [67, p.4]. In this process, the scientist "searches in the reality what contradicts the previous knowledge. (...) The next experience says no to the previous ones. (...) But this no is never definitive for a spirit that knows to dialectize its own principles." [67, p.7] In this sense, Bachelard understands science as an eternally incomplete project. In each step, scientists approach reality by denying the previous experiences. Thus, reality cannot be searched in the pure phenomenon but in the abstract theory that dialogues with it, in the rationalization of the empirical world. In a certain way, it is possible to say that Bachelard defends reality as a construction: "To dialectize the thought is to increase the guarantee of creating scientifically complete phenomena" [67,p. 10]. To Bachelard, reality is only achieved in the theoretical construction, in the abstraction, in the organized phenomenon.

In order to discuss this reality 'construction', Bachelard distinguishes two sorts of reality: the "given real" and the "scientific real" [68]. The given real refers to the sensorial immediate reality, which we experience in the daily life. In the other hand, the "scientific real" is not "given" and it must be accessed or articulated through what Bachelard calls "phenomenotechnique", which is the phenomenon associated to techniques and technologies. For instance, we all have the perception that the Sun turns around Earth (that would be the given real). The challenge of Science is to overcome such first impressions and, by using rigorous methods (such as astronomical observations interpreted in the light of Galilean Physics), to find out that the Earth turns around Sun instead.

Acknowledging that the heliocentric system is part of the "scientific real" means to recognize that this proposition is not evident, but it demands observations, experiments, theories, etc. We may say that its correctness may only be sustained by these scientific elements. Without all of them its validity would be very fragile.

After a new scientific proposition is accepted by the community, it is not necessary to always explicit the original phenomenotechnique: if an astronomer wants to compute the distance of a distant star, he may use the solar parallax without having to discuss the fact that the Earth turns around the Sun in the scientific paper. When it is no longer necessary to make the phenomenotechnique explicit, we may say that the scientific fact became a blackbox [69], meaning that the reasons for its credibility are no longer being discussed. This blackboxing process is extremely necessary and productive for science evolution, otherwise we would have to prove first principles from scratch in every paper - an impossible task.

By managing the blackboxes, scientist can improve scientific knowledge in what may be called chain of reference [70]: basically, they use the previous blackboxes to stablish new steps in the scientific chain producing (discovering) new knowledge. In our example, the heliocentric system was used to compute the distance of a star in the sequence. This could be, thus, used to propose some relation between star distance and some other property, and so on.

When we teach Science, however, introducing scientific facts as totally opaque blackboxes may be misleading for pedagogical purposes. It may seem that "truths" appear as magic, or that only genial ideas have place in Science - what is recognized as the myth of the genius [71]. In this sense, both extreme approaches are problematic: to discuss every fact from scratch is impossible for practical purposes; and not to discuss anything of the "phenomenotechnique" may not be significative for students and it may be epistemologically inadequate.

\subsection{Methodological Discussion}

Our first goal is to discuss how contemporary higher education introductory textbooks present de Broglie's wave particle-duality: do they show the phenomenotechnique or do they omit it? How do they describe de Broglie's proposition?

In order to answer these questions, we performed the following steps. We analyzed de Broglie's original papers about the wave-particle duality with the assistance of specialized literature. We mapped the practices, theories and assumptions used by de Broglie to achieve the wave-particle duality proposition and his equation that relates momentum and wavelength. In section three, we summarize these first results in table 1 and, in the sequence, we use them as analytical categories to interpret the textbooks. The results are summarized in table 2 . Some parts of textbooks are also commented.

\section{De Broglie's 'Phenomenotechnique' and Textbooks analysis}

In this section we focus on explicating de Broglie's original conceptions and the phenomenotechnique he mobi- 
Table 1: Practices, theories and assumptions used by de Broglie

\begin{tabular}{|c|c|}
\hline Practices & $\begin{array}{l}\text { Mathematical derivation (theoretical } \\
\text { physics and not experimental physics) }\end{array}$ \\
\hline \multirow{4}{*}{$\begin{array}{l}\text { Previous theories and } \\
\text { Principles }\end{array}$} & Special Theory of Relativity \\
\hline & Statistical Mechanics \\
\hline & Undulatory Physics \\
\hline & Minimization Principles \\
\hline \multirow[t]{2}{*}{ Previous Models } & Bohr's Atom Model \\
\hline & Einstein's Quantum Hypothesis \\
\hline \multirow[t]{2}{*}{ Assumptions } & $\begin{array}{l}\text { Photons have mass (they are equal to } \\
\text { any other particle) }\end{array}$ \\
\hline & $\begin{array}{l}\text { All particles may be associated to an } \\
\text { undulatory phenomenon in the same } \\
\text { fashion }\end{array}$ \\
\hline
\end{tabular}

lizes (section 3.1) and on analyzing which elements of de Broglie's papers were sustained or erased in contemporary Physics textbooks (section 3.2).

\subsection{Discussion about de Broglie's Phenomenotechnique}

De Broglie's work, as a part of the history of science, participates in the dialectic movement described by Bachelard. It could never be considered the "final truth" but a step in the progress of scientific construction of reality. De Broglie had to deny previous concepts to propose the wave-particle duality. Afterwards, elements of his construction were also denied. These denials, however, can never be considered definitive. Since de Broglie proposed a theoretical work, we may say that he contributed in the process of rationalization or abstraction of the empirical phenomenon and he mobilized an entire network of phenomenotechnique in order to achieve such goal.

Usually, the term "phenomenotechnique" refers to the set of equipment and theories used to describe the "scientific real". De Broglie's work, however, may be labeled as theoretical physics, since it does not deal directly with any observation or experiment. Thus, we may say that the "practice" adopted by de Broglie was to perform mathematical derivations and theoretical constructions.

Since the Scientific Revolution, mathematics play a crucial role in scientific theories, being considered a structural part of it [72]. In theoretical Physics, however, it plays an even more substantial role, since it is the main tool used to articulate knowledge [73].
The privilege of mathematical reasoning as a practice of Physics research has already been adopted by other physicists such as Albert Einstein in the beginning of the XXth century. Through the articulation of Thermostatistics, Wien's Law of Radiation and Electromagnetic Theory, Einstein has proposed that electromagnetic radiation (in the limit of validity of Wien's Law) could be considered as composed by a set of localized quanta, whose energy was proportional to their frequency, what, nowadays, is written as $E=h \nu$ [14]. Initially, de Broglie adhered to Einstein's corpuscular conception about electromagnetic radiation and proposed that quanta have mass [56]. By doing so, de Broglie suggested that Maxwell's Equations are an approximation and not the final expression of Electromagnetic Theory. Also, this assumption demands a modification in the second postulate of the STR. The velocity c must be taken as a limiting velocity for bodies with mass. As quanta may have a very small mass, they must have a speed slightly lower than c (light does not propagate at "speed of light"). Interestingly, de Broglie continues to call c speed of light. The value of photon mass was not suggested by de Broglie in the 1922 paper. However, in other works, values around $10^{-50} \mathrm{~g}$ were speculated [57]. In this sense, we may say that de Broglie's proposition is conflictive with well-stablished Physical Theories - a movement that is called counter-inductive [74], [75] Using Bachelard's framework, de Broglie says "no" to the previous conception of light. Although this assumption is not adopted in Contemporary Physics and there is no empirical evidence of it, this topic was not completely forgotten and it is still possible to find papers that discuss the possibility of photons with mass and its implications [76] In this sense, we may say that de Broglie's assumption was also denied by Physics However, as Bachelard points out, this denial can never be understood as definitive.

As we will see, the assumption of quanta with mass turned out to be very auspicious in de Broglie's theory, since it allowed him to propose a generalized description of reality: quanta, like all particles, have mass - all particles, like quanta, have an undulatory phenomena associated to it. That was exactly what he stablished in 1923:

Let's consider a material body with proper mass $m_{0}$ moving with velocity $\mathrm{v}=\beta \mathrm{c}$ in re-

Table 2: Elements of de Broglie's derivation that are mentioned by textbooks.

\begin{tabular}{lccc}
\hline $\begin{array}{l}\text { Theories and assumptios used } \\
\text { to articulate de Broglie's wave- }\end{array}$ & (Nussenzveig, 2014) & (Halliday et al., 2009) & (Tipler and Llewllyin, 2014 ) \\
particle duality & & Xisberg,1985) \\
\hline STR & $\mathbf{X}$ & \\
Quantum Postulate & & \\
Photon with mass & & $\mathbf{X}$ \\
Undulatory Physics & $\mathbf{X}$ & $\mathbf{X}$ & \\
Statistical Mechanics & Bohr's Model & \\
Minimization Principles & &
\end{tabular}


lation to a fixed observer. According to the principle of inertia of energy, it must have an internal energy equal to $m_{0} c^{2}$. In the other hand, the Principle of Quanta leads to attribute to this energy a periodic phenomenon with frequency $\nu_{0}$ such that $m_{0} c^{2}=h \nu_{0}[57]$

Departing from this conception of wave-particle duality (which is valid to all bodies), de Broglie performed a set of mathematical and theoretical articulations which led him to the famous equation $p=h / \lambda$. In table 1 , we summarize the practices, theories and principles, models and assumptions that de Broglie used - what constituted his "phenomenotechnique".

In the category "practice", we intend to acknowledge the division of science in two main sorts. A scientific work may be empirical or theoretical (or a combination of both). This division relies on the dialectic complementarity proposed by Bachelard about empirical and rational philosophy. An empirical work deals mainly with laboratory trials [69] while a theoretical work privileges the articulation of mathematical equations [75]. The difference between these two sorts of science relies on the practice that the scientist mainly performs.

In the categories theories, principles and models, we acknowledge Max Jammer's [17] discussion on physical theories, which must have three elements: mathematical formalism (the postulates), correspondence rules (which connect the formalism with the empirical results) and interpretations (which include models).

Thus, in the category Theories and Principles we refer to scientific constructions that provide a proper mathematical formalism from which is possible to make direct empirical predictions and interpretations of physical phenomenon. Models, in the other hand, are built upon theories - they do not present a closed mathematical formalism. Finally, in the category "assumption" we inform which are the new propositions about reality that de Broglie assumes in order to perform mathematical articulations.

It is necessary to note that de Broglie's relation was obtained through a relativistic construction (as we show in section 4) and it dialogues with Einstein's original quantization and with the conception of photon with mass to structure de Broglie's duality conception. It does not mean however that this is the only way to derive this equation. A mathematical equation may assume different epistemological status and meanings depending on the theoretical context in which it is developed [72].

To be precise, in the contemporary formulation of Quantum Mechanics, in the context of the second quantization, it is found that the eigen-values of the energy operator of the electromagnetic field are multiples of $\mathrm{h} \nu$ [77] - implying a perspective different from the corpuscular interpretation adopted by Einstein [19], [26].

Moreover, the conception of proper mass is no longer consensual in contemporary presentations of Special Theory of Relativity [78], [79] and de Broglie's relation be- tween momentum and wavelength was obtained in the context of the Principle of Uncertainty without having to perform any relativistic consideration [80]

Not only the equations $E=h \nu$ and $p=h / \lambda$ received new demonstrations and meanings but also the duality conception itself assumed new interpretations and implication along the development of Quantum Physics. More specifically the Complementarity Principle of Copenhagen Interpretation assumed that every quantum system could behave as particle or as wave depending on the experimental set up. More recent studies, however, have shown that in the same experimental set up corpuscular and undulatory properties may be observed - what is called intermediary phenomena [81], [82] Furthermore, contemporarily, Quantum duality is also attached to the study of delayed choice and quantum entanglement [83]

The fact that not only the demonstration but also the interpretation of de Broglie's propositions changed in the articulation of contemporary Quantum Physics Theories should not be seen as if they were incorrect. They are a link in the dialectic chain of science. The validity of his ideas is guaranteed by the network of theories and tools that he mobilized to speak about reality, i.e, his phenomenotechnique [67]. In this sense, all Physics concepts change through time and they can never be considered the final truth - their correctness is always relative to the network they mobilize [70] and the epistemological status of an equation is always dependent of the theoretical construction it belongs to [72]. Thus, by analyzing de Broglie original conceptions and how they changed we may have glimpses about how Theoretical Physics works and how it is possible to achieve important results through counter-inductive movements [74].

\subsection{Textbook Analysis}

The first evident characteristic of the analyzed textbooks is that none of the textbooks proceeds any sort of mathematical derivation. This means that the "practice" used by de Broglie to find the mathematical equation that expresses wave-particle duality is ignored. All textbooks only present the result $\left(\mathrm{p}=\frac{\mathrm{h}}{\lambda}\right)$ and some of them treat it as a postulate - which may be considered an epistemological misclassification [72] In the sequence, we searched whether textbooks, at least, mentioned the elements (theories and assumptions) that de Broglie used. Results are summarized in table 2 .

Halliday et al [63] and Eisberg [61] did not mention any element, which means that they are presenting fully opaque blackboxes. Nussenzveig [64] and Tipler and Llewllyin [62] mention some elements but do not show any sort of mathematical articulation.

Since the phenomenotechnique is ignored, the "scientific real" is treated as "given real" and the wave-particle duality is treated as something evident. To sustain such idea, de Broglie's proposition is considered speculative 
(the genius myth), or treated as a postulate (thus, without derivation) by Nussenzveig:

By analogy with $p=h / \lambda$, de Broglie postulates that the wavelength associated with the (non-relativistic) particle of momentum $\mathrm{p}=\mathrm{mv}$ would be: $\lambda=h / p=h / m v[64, \mathrm{p} .272]$

Halliday et al. also states this conception:

In 1924, the French physicist Louis de Broglie proposed the following line of reasoning: a beam of light is a wave, but it transfers energy and momentum to matter particles only in time events through "packets" called photons. Why cannot a particle beam have the same properties? In other words, why cannot we think of an electron or any other particle as a wave of matter? [63, p.196]

The reduction of de Broglie's proposal to a speculative idea was also sustained by Tipler and Llewellyn:

This suggestion was highly speculative, since there was yet no experimental evidence whatsoever for any wave aspects of electrons or any other particles. What had led him to this seemingly strange idea? It was a "bolt out of the blue," like Einstein's "happy thought" that led to the principle of equivalence [62, p.193].

Eisberg and Resnick [58] also stresses the lack of "reality" in de Broglie's proposal:

In his doctoral thesis, presented in 1924 to the Faculty of Sciences at the University of Paris, Louis de Broglie proposed the existence of matter waves. The thoroughness and originality of his thesis were recognized at once but, because of the apparent lack of experimental evidence, de Broglie's ideas were not considered to have any physical reality. [61, p.56]

Besides of promoting the genius myth, we observe that the textbooks do not explain at all what is meant by wave-particle duality. We are only introduced to the momentum-wavelength relation but no interpretation for it is offered. The textbooks posture towards the scientific knowledge is very common in Science Teaching and may be associated to a mythological narrative instead of a scientific one [71].

\section{An Alternative Didactic Proposal: Revealing the chain of reference of de Broglie's Wave-Particle Duality}

We have discussed that introductory textbooks omit any sort of derivation of de Broglie's ideas and they do not discuss in detail which theories and premises de Broglie's propositions are based on. In the other hand, it is impossible, as we have already commented, to demonstrate every idea from scratch. A midterm solution, however, is possible and it may be interesting for Physics students.

In this section, we propose an alternative didactic narrative: instead of introducing de Broglie's wave-particle duality as something from the "given real", we discuss how it is supported by other theories, models and some assumptions. To do so, we articulated de Broglie's original papers and the specialized literature on it. By doing so, we recognize that we are not introducing the contemporary conception about wave-particle duality or even about de Broglie's relation itself ${ }^{2}$ In this sense, we highlight that this derivation relies on old Quantum Physics and it would not be considered part of the scientific real today (according to Bachelard's framework). Despite of that, we understand that by explicating de Broglie's original arguments, assumptions and practices, and the specialized literature interpretation, we may contribute to a more meaningful education, one that not only introduces physical concepts but that allows the student to have at least a glimpse of how science works and evolves.

As we intend to propose a didactic narrative, we did not follow all de Broglie's steps in the historical trajectory, but we present (in agreement with de Broglie's proposal) an organized demonstration of the relation $p=\frac{h}{\lambda}$. Specificically, we show that this equation may be derived from Special Theory of Relativity (STR) and from what de Broglie calls Einstein's Quantization Hypothesis (QH) with the addition of two assumptions (which make clear what de Broglie understands as wave-particle duality) and of wave theory. After deriving de Broglie's relation, we discuss how it provides the continuation of new experiments and theories. Our approach can be schematically represented as a "chain of reference" (Figure 1):

We will not derive the theoretical premises. They will be only presented (in the sense we have been used, they are still blackboxes). We will show how their articulation promote the derivation of $\mathrm{p}=\frac{\mathrm{h}}{\lambda}$.

\subsection{Theoretical premisses}

In order to propose a didactic approach, it is possible to derive de Broglie's relation using STR and QH. We will present the main relations necessary for such derivation.

\section{i) Special Theory of Relativity (STR)}

Originally, STR was proposed by Albert Einstein in 1905, using two postulates. The first postulated stated that the same Laws of Electrodynamics and Optics are valid for all references in which the Mechanics equations are valid. The second postulate stated that light always propagates in the empty space with a definite velocity c, which is independent of the state of motion of the emitting body.

\footnotetext{
2 About the contemporary conception on this issue, the reader may consult references offered in section 3.1 .
} 


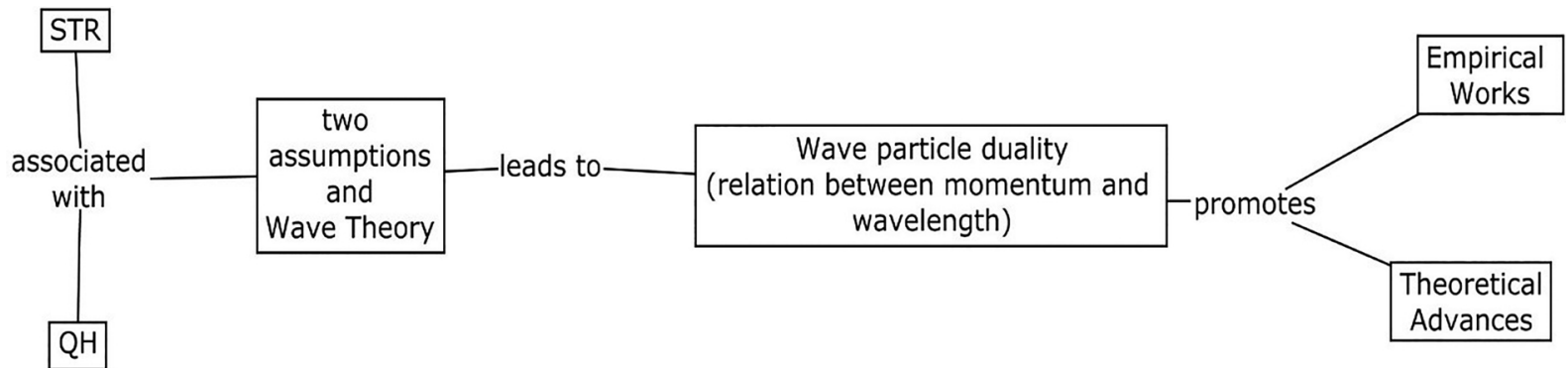

Figure 1: Chain of reference to teach de Broglie's wave particle duality.

From these two postulates, all the other famous consequences of STR (contraction of space, dilation of time, etc.) can be derived. We will not present such derivations, as this has already been done exhaustively in the literature and can be found in Basic Physics books [64]. We will only present the results that are used in the next section.

\section{a) Lorentz's Transformation and Relativistic Frequency}

It is possible to show that in order to satisfy the second postulate, Galileo's Principle of Relativity must be renewed. A linear transformation of spacetime coordinates that satisfies this condition is called Lorentz Transformations, which relate the three spatial coordinates and the time coordinate of the same event in two frames ( $\mathrm{S}$ 'and $\mathrm{S}$ ) when $\mathrm{S}$ ' moves with a velocity $\mathrm{v}$ with respect to $\mathrm{S}$ along the $\mathrm{X}$-axis:

$$
\begin{aligned}
& x^{\prime}=\gamma(x-v t) \\
& y^{\prime}=y \\
& z^{\prime}=z \\
& t^{\prime}=\gamma\left(t-\frac{v x}{c^{2}}\right)
\end{aligned}
$$

In which,

$$
\gamma=\frac{1}{\sqrt{\left(1-\beta^{2}\right.}}
$$

And

$$
\beta=\frac{v}{c}
$$

From the Lorentz equations it is possible to obtain the time dilation equation. Let us consider as the proper interval of time $\left(t_{0}\right)$ the measure of time between two events in the same position of space. In this case, equation 1.4 takes the following form:

$$
t^{\prime}=\gamma t_{0}=\frac{t_{0}}{\sqrt{\left(1-\beta^{2}\right.}}
$$

That is, the measure of time performed in reference frame that is not at rest in relation to the studied phenomenon $\left(\mathrm{S}^{\prime}\right)$ is greater than that performed in the reference at rest $(\mathrm{S})$. On the other hand, if we compare the frequencies of two clocks in the different references, we obtain the following relations:

$$
\frac{1}{t_{0}}=\frac{\gamma}{t^{\prime}} \rightarrow \nu^{\prime}=\frac{\nu_{0}}{\gamma}=\nu_{0} \sqrt{1-\beta}
$$

The frequency measured in $S^{\prime}$ is smaller than the frequency measured in $\mathrm{S}$.

\section{b) Relativistic Momentum}

In STR, the momentum (p) must also be corrected, so that it can be expressed as the classical momentum times the Lorentz factor $(\gamma)$ :

$$
p=\frac{m_{0} v}{\sqrt{1-\beta}}
$$

It should be noted that the expression of the relativistic momentum tends to the expression of the classical momentum, when the velocity factor $(\beta)$ tends to zero. On the other hand, for particles with velocity close to the speed of light, relativistic expression becomes necessary.

\section{c) Relation between energy and mass}

It is also possible to relate the mass value of a particle with energy associated with it. When this relation is made at the reference where the particle is at rest one obtains:

$$
E_{0}=m_{0} c^{2}
$$

When the energy is measured in another reference, one must also take into account the relativistic correction 3

$$
E=m c^{2}=\frac{m_{0} c^{2}}{\sqrt{1-\beta^{2}}}
$$

\section{d) Relativistic Kinetic Energy}

For an observer in the reference where the body is at rest, the kinetic energy of the body is zero. In another reference, however, the kinetic energy must be the total energy of the body, excluding the energy associated with

\footnotetext{
3 We highlight that we are using the notation of rest mass and relativistic mass. Such notation is not original from Einstein's work and can imply conceptual problem [79], [84]. Nevertheless, we use it because de Broglie adopts such formulation.
} 
the resting mass (considering that there are no interaction potentials involved). In this case, the relativistic expression of kinetic energy can be expressed as:

$$
W=m_{0} c^{2}\left(\frac{1}{\sqrt{1-\beta^{2}}}-1\right)
$$

\section{e) Relationship between energy, momentum and mass}

Combining equations (6), (7) and (8), one can relate energy, momentum and rest mass:

$$
E^{2}=p^{2} c^{2}+m_{0}^{2} c^{4}
$$

Equations 1-10 synthesize the expressions of STR that are necessary to understand the proposal of Louis de Broglie. Nonetheless, it is necessary to use the equation that relates energy and frequency in Quantum Theory.

\section{ii) Einstein's Quantization Hypothesis}

The equation that relates energy (E) and frequency $(\nu)$ in Quantum Theory was obtained in independent ways by Planck and Einstein (1905a)

$$
E=h \nu
$$

The meaning of such an expression also diverged between the two authors. Whereas for Planck, the equation expressed the energy of the oscillators within matter, for Einstein, the relation refers to the energy of the quanta of light. For Einstein, each quantum is a corpuscle with position defined in space and with energy $h \nu$. However, how a corpuscle can be associated with a frequency was not explained by Einstein ${ }^{5}$ As we shall see, not only de Broglie redefines the meaning of the mathematical expression, but also explains the relation between particle and wave.

\subsection{Deriving Louis de Broglie's relation between momentum and wavelength}

Having in mind the concepts defined in the scope of STR and Quantum Theory, it is possible to understand the quantum proposal of Louis de Broglie as well as the innovations that it brings. In this work, we present two theses that synthesize this conception. As we shall see, both theses progress in order to provide a generalized theory for quantum objects. Instead of needing a theory to speak of electromagnetic radiation and another one to speak of electrons, for example, de Broglie's proposal places all

\footnotetext{
4 We are not adopting the original notation, but the popular notation nowadays.

${ }^{5}$ In the 1905 article, Einstein proposed that the wave properties of radiation appear only in cases where the measured magnitude refers to a statistical average of a set of quanta [14]. In 1909, Einstein adopts a different view, speculating that radiation should be composed of corpuscles and an associated vector field [85]. However, he never managed to formalize such an insight [15].
}

quantum elements in ontological equality. According to the conception developed by the special theory of relativity, as we mentioned, light cannot have mass. Even today, the hegemonic view agrees with such a conception and establishes a distinction between what would be quantum description for classical particles (Schrodinger equation), relativistic particles (Klein-Gordon equation), spin particles (Dirac equation) and photons (Second Quantization). As we will see, the original proposal of de Broglie would not agree with such distinctions.

The first thesis we present extends the notion of quantum as a particle (and therefore ontologically identical to other particles of matter), and the second thesis associates an undulatory phenomenon to all the particles, generalizing the notion that every quantum entity is composed of a particle and an associated wave. Thus, we highlight three elements of de Broglie's Theory: first, it refers to all beings in the world; second, it is a symmetrically dual theory (it is not that there are particles that behave as matter and waves that behave as particles: all beings are composed of particles and oscillatory phenomena); third, it is a relativistic theory.

\section{Assumption 1. Quanta are atoms (indivisible particles) of light with rest mass different from zero. All quanta are identical at rest}

Following de Broglie [56], in order to measure how the velocity of light atoms is affected by the existence of mass, we can derive a relation from equations (8) and (11):

$$
\frac{m_{0} c^{2}}{\sqrt{1-\beta^{2}}}=h \nu
$$

By squaring both sides of the equation (12) and using (3), we have:

$$
\frac{m_{0}^{2} c^{4}}{1-\beta^{2}}=\frac{m_{0}^{2} c^{4}}{1-\frac{v^{2}}{c^{2}}}=h^{2} \nu^{2}
$$

When isolating velocity, we obtain:

$$
v^{2}=c^{2}-\frac{m_{0}^{2} c^{6}}{h^{2} \nu^{2}}
$$

And

$$
v=\sqrt{c^{2}-\frac{m_{0}^{2} c^{6}}{h^{2} \nu^{2}}}
$$

According to equation (15), the greater the frequency of radiation, the greater its speed. Figure 2 shows how the velocity parameter behaves in relation to the frequency of the electromagnetic radiation. It is observed that it would be necessary to produce very low frequency electromagnetic radiation so that its velocity be significantly perceptible below the speed of light.

With regard to thesis 1 , it is observed that it is not yet a dual proposal by itself. It simply develops Einstein's proposal to understand quantum as a corpuscle. 


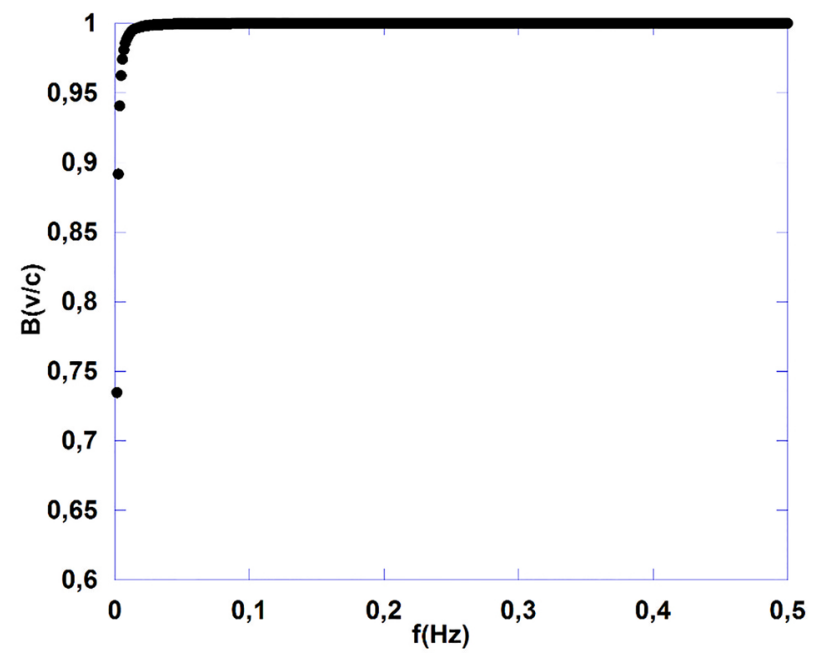

Figure 2: Velocity parameter for photons in relation to their frequency.

De Broglie takes such a conception to its ultimate consequences [15], endowing mass to quanta like any other "material" particle. This creates a first symmetry between quanta and other particles. The issue solved by thesis two is how to associate such particles to an undulatory nature.

In order to propose such a relation, we can start from equation 14 , in the case where we are in the reference frame of the atom of light (which is possible, as it has mass). In this case, there is a frequency associated with the rest energy of the light atom:

$$
h \nu=m_{0} c^{2}
$$

That is, at rest, the light atom has an oscillatory motion whose frequency can be associated with its rest mass. As we have proposed, however, there is nothing that distinguishes atoms of light from other forms of particle. In addition, de Broglie had theoretical reasons to infer that light and matter may be identical. Both propagate obeying a principle of minimization. Light follows the principle of Fermat, and particles the principle of minimization of action. In 1924, de Broglie showed that both can be unified in a single formalism [58]. In addition, Bohr's electron quantization rule bears great resemblance to the quantization of the vibrational modes of a standing wave, which was discussed by de Broglie in 1923 [57]. Finally, Stark had already calculated the frequency associated with the rest mass of the electron in 1907 using the relation (14) [15]
Assumption 2. Every particle is associated with a periodic phenomenon. In the reference where the particle is at rest, such phenomenon has frequency $\nu_{0}$, so that $\mathrm{h} \nu_{0}$ corresponds to the rest energy associated with the particle. In another frame (where the velocity of the particle is different from zero) such a phenomenon is interpreted as a group of phase waves with similar frequency.

It should be noted that the quanta have already been considered by de Broglie as particles. So, thesis 2 refers to all bodies (without distinction between radiation and matter). However, by means of association of a frequency $\nu_{0}$ with the mass of any particle, de Broglie generalizes equation (11), which was obtained strictly in the context of quanta. The equation by Planck and Einstein then starts to be used for every quantum object.

To analyze consistency and implications of the second thesis 6 , let us assume a periodic movement in the reference where the particle is at rest (such as a harmonic oscillation):

$$
\Psi\left(t_{0}\right)=A \cos \left(\omega_{0} t_{0}\right)
$$

In another reference frame, where the particle is seen as moving with velocity $v$, by using equation (1.4), we have that the oscillatory motion is described by

$$
\Psi(x, t)=A \cos \left[\omega_{0} \gamma\left(t-\frac{v x}{c^{2}}\right)\right]
$$

Comparing this equation with the function of a wave

$$
\Psi(x, t)=A \cos (\omega t-k x)
$$

The following relations are reached:

$$
\omega=\omega_{0} \gamma \text { and } k=\frac{\omega_{0} \gamma v}{c^{2}}
$$

One can also calculate the velocity with which such a wave propagates:

$$
V=\frac{\omega}{k}=\frac{c^{2}}{v}=\frac{c}{\beta}
$$

Since $\beta$ is always less than 1 , we have that the velocity of the wave is greater than the speed of light. According to the Special Theory of Relativity, such a wave cannot carry energy (since this transmission speed would demand an infinite energy). Because of this, de Broglie called it, at first, a fictitious wave, abandoning that term in subsequent articles [15]. From equation (20) we can still express the angular frequency of the motion in terms of the velocity parameter:

$$
\omega=\frac{\omega_{0}}{\sqrt{1-\beta^{2}}}
$$

${ }^{6}$ We follow, throughout this argument, the original article [57] as well as considerations by Roberto Martins e Rosa [15] and Brown \& Martins [59]. 
Comparing this expression with equation (5), we observe that the frequency of the associated wave is not transformed as the clock frequency. On the contrary, the Lorentz factor appears inverted in both equations. The expression (21), though, is transformed in the same way as the energy of the particle (Equation 8):

$$
\begin{aligned}
E & =h \nu=\frac{h \omega}{2 \pi}=\frac{h \omega_{0}}{2 \pi \sqrt{1-\beta^{2}}}=\frac{h \nu_{0}}{\sqrt{1-\beta^{2}}} \\
& =\frac{m_{0} c^{2}}{\sqrt{1-\beta^{2}}}
\end{aligned}
$$

And may relate frequency with mass and the velocity parameter:

$$
\nu=\frac{m_{o} c^{2}}{h \sqrt{1-\beta^{2}}}
$$

Thus, we can say that the frequency of the wave associated with the particle is equivalent to the frequency associated with the energy of the particle for any inertial reference. The fact that energy and frequency satisfy the same transformation rule is a positive point for the consistency of the theory. However, one must still solve the problem of why the frequency transformation does not follow the usual frequency transformation.

For this, it should be noted that in thesis 2, it is stated that the particle is not associated with a single wave, but with a group of waves with similar frequencies. We will show that, although each wave has velocity $c / \beta$, the velocity of the group is $\beta c$ (the velocity of the particle) and although the frequency of each phase wave does not vary according to the frequency transformation, the beat frequency of the group does. To demonstrate this, we use the expression of the group velocity (U) used by de Broglie 7 .

$$
\frac{1}{U}=\frac{d\left(\frac{\nu}{V}\right)}{d \nu}
$$

Isolating $\beta$ in equation (24), we obtain:

$$
\beta=\frac{\sqrt{\nu^{2} h^{2}-m_{0}^{2} c^{4}}}{h \nu}
$$

Replacing (26) in (21):

$$
V=\frac{c}{\beta}=\frac{c h \nu}{\sqrt{\nu^{2} h^{2}-m_{0}^{2} c^{4}}}
$$

We can now calculate $\frac{\nu}{V}$

$$
\frac{\nu}{V}=\nu \frac{\sqrt{\nu^{2} h^{2}-m_{0}^{2} c^{4}}}{c h \nu}=\frac{\sqrt{\nu^{2} h^{2}-m_{0}^{2} c^{4}}}{c h}
$$

Taking the derivative of (28) concerning $\nu$ and replacing it (25):

$$
\frac{1}{U}=\frac{d\left(\frac{\nu}{V}\right)}{d \nu}=\frac{h \nu}{c \sqrt{\nu^{2} h^{2}-m_{0}^{2} c^{4}}}=\frac{1}{\beta c}
$$

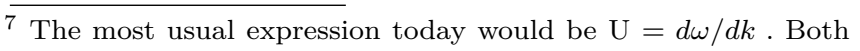
expressions are equivalent.
Which ultimately provides,

$$
U=\beta c
$$

To calculate the beat frequency, one can start from (22):

$$
\nu=\frac{\nu_{0}}{\left(1-\beta^{2}\right)^{\frac{1}{2}}} \rightarrow \nu^{2}=\frac{\nu_{0}^{2}}{\left(1-\beta^{2}\right)}=\frac{\nu_{0}^{2}}{\left(1-\frac{c^{2}}{V^{2}}\right)}
$$

Still, we can use the relation valid for all waves $V=\lambda \nu$ in (31):

$$
\frac{V^{2}}{\lambda^{2}}\left(1-\frac{c^{2}}{V^{2}}\right)=\nu_{0}^{2}
$$

Isolating $V^{2}$ :

$$
V^{2}=c^{2}+\nu_{0}^{2} \lambda^{2}
$$

Finally, we can determine the beat frequency [59]:

$\nu^{\prime}=\frac{\partial V}{\partial \lambda}=\frac{\nu_{0}^{2} \lambda}{V}=\frac{\nu_{0}^{2} \lambda}{\lambda \nu}=\frac{\nu_{0}^{2}}{\frac{\nu_{0}}{\left(1-\beta^{2}\right)^{\frac{1}{2}}}}=\nu_{0}\left(1-\beta^{2}\right)^{\frac{1}{2}}$

This gives an expression that is transformed in the same way of equation (5). Further on, we can explain wave properties associated to the particles anticipated in the thesis 2 . We related wave characteristics to phase and group in Table 3.

Once the internal consistency of de Broglie's proposal is guaranteed, we can keep on advancing in the construction of our theoretical knowledge about this wave-particle system. Let us investigate how the particle's momentum relates to the associated wavelength using the proposal of Brown and Martins [59]. We start from equation (8):

$$
E^{2}\left(1-\frac{v^{2}}{c^{2}}\right)=m_{0}^{2} c^{4}
$$

We can rearrange the terms as:

$$
E^{2}-m_{0}^{2} c^{4}=\frac{E^{2} v^{2}}{c^{2}}
$$

Still, we can combine $V=\lambda \nu$ and $V=c^{2} / v$ to write $v$ as a function of wavelength and frequency:

$$
v=\frac{c^{2}}{\lambda \nu}
$$

Table 3: Wave characteristics related to phase and group.

\begin{tabular}{lll}
\hline Characteristics & $\begin{array}{l}\text { Phase velocity of a } \\
\text { Wave }\end{array}$ & $\begin{array}{l}\text { Group velocity of a } \\
\text { Wave }\end{array}$ \\
\hline Velocity & $V=\frac{c}{\beta}$ & $U=\beta c=v$ \\
$\begin{array}{l}\text { Relation with } \\
\text { Energy }\end{array}$ & $E=\frac{h \nu_{0}}{\sqrt{1-\beta^{2}}}$ & \\
$\begin{array}{l}\text { Transformation of } \\
\text { the Frequency }\end{array}$ & $\nu=\frac{\nu_{0}}{\left(1-\beta^{2}\right)^{1 / 2}}$ & $\nu^{\prime}=\nu_{0}\left(1-\beta^{2}\right)^{1 / 2}$ \\
\end{tabular}


Substituting (37) into (36) and using (10) and (11):

$$
p^{2} c^{4}=\frac{h^{2} \nu^{2} c^{4}}{\lambda^{2} \nu^{2}}
$$

By simplifying the expression, we get:

$$
p=\frac{h}{\lambda}
$$

Such an expression relates the moment of any particle to the wavelength associated to it. In this context, $\lambda$ is usually called the de Broglie wavelength. It should be noted that it is valid for all particles (photons, electrons, protons, etc.) and was obtained from a relativistic derivation. The same relation could be obtained, only for photons, assuming the notion of zero mass, using (10) and (11). However, this is not what de Broglie did. He came to this relation for all particles (and not just for photons) without having to assume zero mass for any particle and without limiting its derivation to the non-relativistic case. In this process, however, we assume the existence of immaterial waves with associated supraliminal velocity.

We stress that the previous derivation uses only differential calculus as mathematical framework - which can be easily used by students from fourth semester of Physics and Engineer courses.

\section{Using de Broglie's Wave-Particle Duality to continue the chain of reference}

As we have discussed in section 2, science evolves in chain. de Broglie's relation was obtained based in previous theories and, symmetrically, it works as base for new works. As important as deriving de Broglie's equation is to stress the empirical studies that corroborate it and the theoretical advances that it allows. In this section, we sketch possibilities for the Physics teacher to use in his classroom:

\section{a) Empirical Works that corroborate de Broglie's proposition}

The dual conception of de Broglie symmetrizes all components of reality. In the same sense that photons are particles that interfere, all particles should also interfere. In this sense, using the correct parameters it is possible to observe other particles performing undulatory phenomena.

This is exactly the kind of empirical corroboration that de Broglie's proposition has obtained [26]. After deriving de Broglie's equation, we suggest that students may be invited to read key papers that discuss interference of electrons [86], neutrons [87], atoms [88] and Bose condensates [89]. By doing so, the professor allows the student to be in touch with the specialized literature an important activity that has been commonly neglected by Science Education [90].

\section{b) Theoretical advances from de Broglie's proposition}

Another contribution of de Broglie's work may be found in the continuation of the chain of Theoretical Physics. In this section, we show how we can use it to "suggest" a wave equation for Quantum Mechanics.

Let's assume that the set of waves associated to a particle may be represented by exponential functions as it is usual in wave mechanics. In order to make the easiest approach, let's take only one of these exponential functions:

$$
\Psi=e^{i(k x-\omega t)}
$$

Using Einstein's and de Broglie's equations, we may relate energy with angular frequency and momentum with wavenumber.

$$
E=\hbar \omega \quad \text { and } \quad p=\hbar k
$$

We also know that energy, may be written as

$$
E=\frac{p^{2}}{2 m}+V
$$

From (40) and (41), we obtain that

$$
E \Psi=i \hbar \frac{\partial}{\partial t} \Psi \quad \text { and } \quad \frac{p^{2}}{2 m} \phi=-\frac{\hbar^{2}}{2 m} \frac{\partial^{2} \Psi}{\partial x^{2}}
$$

Combining (42) and (43), we may suggest Schrödinger Equation:

$$
i \hbar \frac{\partial}{\partial t} \Psi=-\frac{\hbar^{2}}{2 m} \frac{\partial^{2} \Psi}{\partial x^{2}}+V \Psi
$$

Observe that de Broglie's relation is relativistic while the energy balance expression in equation (42) is nonrelativistic, which makes Schrodinger Equation non-relativistic. If we use the same strategy in association with the relativistic energy expression- equation (10) in this paper we obtain Klein-Gordon equation:

$$
\frac{1}{c^{2}} \frac{\partial^{2} \Psi}{\partial t^{2}}-\frac{\partial^{2} \Psi}{\partial x^{2}}+\frac{m^{2} c^{2}}{\hbar^{2}} \Psi=0
$$

\section{Final Remarks}

In this paper, we have discussed the difference between "given real" and "scientific real", stressing that the validity of all scientific facts must be supported by a set of techniques, technologies and theories (what Bachelard calls "phenomenotechnique"). After mapping the practices, tools and assumptions used by de Broglie to propose the wave-particle duality, we examined which of these elements were still present in contemporary introductory textbooks.

Our main results showed that textbooks omit almost all elements used by de Broglie and they do not perform any mathematical derivation, presenting it as a "blackbox". 
This pedagogic orientation may be strongly associated to an instrumentalist perspective, which became hegemonic in the post-war period [91], when the focus was not to discuss the theoretical grounds of physical theories but to allow the technical formation of engineers and scientist that would contribute for technological development. That is one of the reasons why contemporary Quantum Physics textbooks overemphasize the instrumental application of formulae and do not discuss the theories in detail.

We understand that this pedagogic orientation, as interesting as someone can find it to be, is not necessary any longer. We comprehend that a scientific formation encompasses not only the development of the capacity of manipulating equations but also the understanding of the articulation and of the meaning of these equations. Mathematics is a structural part of Physics [72] and it cannot be treated as a simple tool that we use when it is applicable. To derive an equation such as the wavelengthmomentum relation allows us to understand what it means and what it is speaking about. Also, we defend that a scientific education should be concerned in showing how science works and how it achieves its results more than merely informing the student about the results.

In order to present an alternative didactic approach, we proposed a chain of reference presentation, stressing the elements that supported de Broglie's proposition according to [15], [56]-[60]. We have shown that using Special Theory of Relativity and the Quantum Hypothesis in association with the assumptions that quanta have mass and that all particles may be associated to a periodic phenomenon; it is possible to derive de Broglie's relation between momentum and wavelength. In the end, we outlined empirical works that corroborate de Broglie's proposition and we showed how it can be used to suggest the articulation of Schrodinger's and Klein-Gordon's equations.

\section{References}

[1] L. L. Landau and E. Lifchitz, Théorie du champ. (Mir, Moscou, 1966).

[2] J. J. Sakurai, Advanced Quantum Mechanics. (Albert Whitman \& Company, 2013).

[3] R. J. Glauber, Phys. Rev. Lett. 10, 84 (1963).

[4] R. J. Glauber, Phys. Rev. 130, 2529 (1963).

[5] F. Benatti, Dynamics, in Information and Complexity in quantum Systems, (Dordrecht Springer, Netherlands, 2009).

[6] S. Vinjanampathy and J. Anders, Contemp. Phys. 57, 545 (2016).

[7] R. P. Woodard, Reports Prog. Phys. 72, 126002, (2009).

[8] S. W. Young, Nuclear Magnetic Ressonance: Basic Principles. (Raven Press, New York, 1984).

[9] A. R. G. L.Hornyak, H. F. Tibbals, J. Dutta, Introduction to Nanoscience. (CRC Press, New York, 2008).

[10] J. M. Marin, Eur. J. Phys. 30, 807, (2009).
[11] O. Freire, The Quantum Dissidents: Rebuilding the Foundations of Quantum Mechanics (1950-1990) (Springer, Berlin, 2015).

[12] R. Feynman, R. Leighton, and M. Sands, Feynman's Lectures on Physics. (Pasadena, California Institute of Technology, 2013).

[13] A. Einstein, in Albert Einsteins - The Swiss Years: Writings, 1900-1909. Edited by John Stachel, David C. Cassidy, Jürgen Renn, and Robert Schulmann, (1990).

[14] A. Einstein, Ann. Phisik, 17, (1905).

[15] R. de A. Martins and P. S. Rosa, História da teoria quântica - a dualidade onda-partícula, de Einstein a De Broglie. (Livraria da Física, São Paulo, 2014).

[16] O. Pessoa Jr., Conceitos de Física Quântica. Livraria da Física, São Paulo, 2003.

[17] M. Jammer, The Philosophy of Quantum Physics. (New York, John Wiley and Sons, 1974).

[18] N. W. Lima, B. B. De Souza, C. J. de H. Cavalcanti, and F. Ostermann, Rev. Bras. Pesqui. em Educ. em Ciências, 18, 365, (2018).

[19] N. W. Lima, E. Antunes Jr, C. J. de H. Cavalcanti, and F. Ostermann, Ensen. las Sci., vol. especial, 1953, (2017).

[20] E. Schrodinger, Collected Papers on Wave Mechanics. (London, Backie \& Son Limited, 1928).

[21] E. Schrodinger, Ann. der Phys. 4, 257 (1927).

[22] M. Born, Zeitschrift fur Phys., 37, 863 (1926).

[23] N. Bohr, Nature, 121, 580 (1928).

[24] A. Hobson, Am. J. Phys., 81, 211 (2013) .

[25] I. Silva, Revista Brasileira de Ensino de Física , 37, 4204 (2015).

[26] G. Greenstein and A. G. Zajone, The Quantum Challenge - Modern Research on the Foundation of Quantum Mechanics. (Jones and Bartlett Publishers, Sudbury, 2006).

[27] Y. W. Cheong and J. Song, Science \& Education, 23, 1011 (2014)

[28] G. Matteucci, Eur. J. Phys. 733 (2011).

[29] E. Marshman and C. Singh, European Journal of Physics 39 (2017)

[30] N. W. Lima, F. Ostermann, and C. J. de H. Cavalcanti, Cad. Bras. Ensino Fúsica, 435 (2017).

[31] D. Dehlinger and M. W. Mitchell, Am. J. Phys. 70, 898 (2002).

[32] D. Dehlinger and M. W. Mitchell, American Journal of Physics 70, 898 (2002).

[33] T. L. Dimitrova and A. Weis, American Journal of Physics 76, 137 (2008).

[34] E. J. Galvez, Am. J. Phys. 78 (2010).

[35] E. J. Galvez, C. H. Holbrow, M. J. Pysher, J. W. Martin, N. Courtemanche, L. Heilig, and J. Spencer, American Journal of Physics 73, 127 (2005).

[36] C. H. Holbrow, E. Galvez, and M. E. Parks, Am. J. Phys., 70, 260 (2002).

[37] A. Goff, Am. J. Phys., 74, 962 (2006).

[38] K. Jacobs and H. M. Wiseman, Am. J. Phys. 73, 932 (2005).

[39] E. M. and C. Singh, Eur. J. Phys. 37, 24001 (2016).

[40] J. S. Netto, C. J. de H. Cavalcanti, and F. Cad. Bras. Ensino Física, 35, 185 (2018).

[41] C. Ferrari and B. Braunecker, Am. J. Phys., 78, 792 (2010). 
[42] M. Gordon and G. Gordon, Phys. Educ., 45, 372 (2010).

[43] A. Kohnle, M. Douglass, T. J. Edwards, A. D. Gillies, C. A. Hooley, and B. D. Sinclair, Eur. J. Phys., 31, 1441, (2010).

[44] S. B. McKagan, K. K. Perkins, M. Dubson, C. Malley, S. Reid, R. LeMaster, and C. E. Wieman, Am. J. Phys., 76, 406, (2008).

[45] R. Müller and H. Wiesner, Am. J. Phys., 70, 200, (2002).

[46] C. J. H. Cavalcanti, F. Ostermann, N. W. Lima, and J. S. Netto, Eur. J. Phys., 38, (2017).

[47] N. W. Lima, C. J. de H. Cavalcanti, F. Ostermann. Caderno Brasileiro de Ensino de Física, 34, 435 (2017).

[48] N. W. Lima, E. Antunes Jr, C. J. de H. Cavalcanti, and F. Ostermann, Ensen. las Sci., vol. especial, 1947, 2017.

[49] A. Henke and D. Höttecke, Sci. Educ., 24, 349 (2015).

[50] D. R. Geelan, Res. Sci. Educ., 27, 553, (1997).

[51] T. Kuhn, The structure of Scientific Revolutions, (University of Chicago Press, Chicago, 1996).

[52] T. Kuhn, The Essential Tension: Selected Studies in Scientific Tradition and (University of Chicago Press, Chicago, 1977).

[53] M. Badino and J. Navarro, A History of Quantum Physics through Textbooks. (Edition Open Access, Berlin, 2013).

[54] B. Bensaude-Vincent, Br. J. Hist. Sci., 23, 435, 1990.

[55] D. Kaiser, Pedagogy and the practice of science. (The MIT Press, Cambridge, 2006).

[56] L. de Broglie, J. Phys. Radium, 3, 22 (1922).

[57] L. de Broglie, Comptes Rendus, 177, 507 (1923).

[58] L. de Broglie, Philos. Mag., 47, 446 (1924).

[59] H. R. Brown and R. de A. Martins, Am. J. Phys., 52, 1130 (1984).

[60] J. S. and W. Kuhn, Eur. J. Phys., 6, 176, (1985).

[61] R. Eisberg and R. Resnick, Quantum Physics of Atoms, Molecules, Solids, Nuclei and Particles. (John Wiley and Sons, New York, 1985).

[62] P. A. Tipler and R. A. Llewellyn, Modern Physics. W. H. (Freeman and Company, New York, 2012).

[63] D. Halliday, R. Resnick, and J. Walker, Fundamentos de Física: ótica e física moderna. (LTC, Rio de Janeiro 2009).

[64] H. M. Nussenzveig, Curso de Física Basica. (Editora Blucher, Rio de Janeiro, 2004).

[65] A. Chalmers, O que é ciência afinal. (Editora Brasiliense, São Paulo, 1993).

[66] I. Niniluoto, Critical Scientific Realism. (Oxford University Press, Oxford, 1999).

[67] G. Bachelard, A Filosofia do Não. (Cultural, São Paulo, 1978).

[68] G. Bachelard, The new Scientific Spirit. (Beacon, Boston , 1985).

[69] B. Latour, Science in Action: How to follow scientists and engineers through society. (Harvard University Press, Cambridge ,1988).

[70] B. Latour, Pandora's Hope: Essays on the reality of Science Studies. (Harvard University Press, Cambridge, 1999).

[71] D. Allchin, Sci. Educ., 13, 179 (2004).

[72] R. Karam and O. Krey, Sci. Educ., 24, 661 (2015).

[73] N. W. Lima, T. Venturini, F. Ostermann and C. J. deH. Cavalcanti. arXiv:1811.04509 (2018).
[74] P. Feyerabend, Against the Method. (Verso, London, 1995).

[75] N. W. Lima, T. da S. Peron, and A. G. Moraes, in 15th Internation History and Philosphy of Science Teaching, (Dover Publications inc, New York, 2019) p. 239.

[76] A. S. Goldhaber and M. M. Nieto, Rev. Mod. Phys., 82, 939 (2010).

[77] M. Beck, Quantum Mechanics: Theory and Experiment. (Oxford University Press, Oxford, 2012).

[78] F. Ostermann and T. Ricci, Cad. Bras. ensino Física, 21, 83 (2004).

[79] F. B. Kneubil, Rev. Bras. Ensino Fisica, 40 (2018).

[80] J.-M. Schwindt, Conceptual Basis of Quantum Mechanics. (Springer, Berlin, 2016).

[81] T. Qureshi, American Journal of Physics 84, 517 (2016).

[82] B. Englert, Phys. Re, 77, 154 (1996).

[83] X. Ma, J. Kofler, and A. Zeilinger, Rev. Mod. Phys., 88, 15005 (2016).

[84] F. Ostermann and T. Ricci, Cad. Bras. Ensino Física, 21, 83 (2004).

[85] A. Einstein, Rev. Bras. Ensino Física, 27, 77 (2005).

[86] A. Tonomura, J. Endo, T. Matsuda, T. Kawasaki, and H. Ezawa, Am. J. Phys., 57, 117, (1989).

[87] R. Gähler and A. Zeilinger, Am. J. Phys., 59, 316 (1991).

[88] O. Carnal and J. Mlynek, Phys. Rev. Lett., 66, 2689 (1991).

[89] M. R. Andrews, C. G. Townsend, H.-J. Miesner, D. S. Durfee, D. M. Kurn, and W. Ketterle, Science, 275, 637 (1997).

[90] N. W. Lima, F. Ostermann, and C. J. de H. Cavalcanti, Cad. Bras. Ensino Física, 35, 367 (2018).

[91] D. Kaiser, K. Ito, and K. Hall, Soc. Stud. Sci., 34, 879 (2004). 